\title{
Impact of Snail and E-cadherin expression in pancreatic neuroendocrine tumors
}

\author{
KEIICHI YONEMORI ${ }^{1}$, HIROSHI KURAHARA ${ }^{1}$, KOSEI MAEMURA ${ }^{1}$, YUKO MATAKI $^{1}$, \\ MASAHIKO SAKODA ${ }^{1}$, SATOSHI IINO ${ }^{1}$, SHINICHI UENO ${ }^{2}$, HIROYUKI SHINCHI ${ }^{3}$ and SHOJI NATSUGOE $^{1}$ \\ Departments of ${ }^{1}$ Digestive Surgery, Breast and Thyroid Surgery, ${ }^{2}$ Clinical Oncology, and ${ }^{3}$ Health Sciences, \\ Graduate School of Medical Sciences, Kagoshima University, Kagoshima 890-8520, Japan
}

Received May 30, 2016; Accepted March 7, 2017

DOI: $10.3892 / \mathrm{ol} .2017 .6306$

\begin{abstract}
Pancreatic neuroendocrine tumors (PNETs) are a rare type of malignancy with a prognosis that is relatively good, compared with that of pancreatic adenocarcinoma. However, a number of patients with PNETs have distant metastasis and a less favorable prognosis. Epithelial-mesenchymal transition (EMT) is essential for tumor progression and metastasis. Snail and E-cadherin serve key roles in the process of EMT in numerous tumor types, including gastric and pancreatic adenocarcinoma. However, the role of EMT in PNETs remains unclear. The aim of the present study was to investigate the significance of Snail and E-cadherin expression in PNETs. Tissue samples were obtained from 40 resected PNETs. The expression of Snail and E-cadherin was evaluated in the primary tumors using immunohistochemical staining. The association between protein expression and patient clinicopathological features was then analyzed. High and low Snail expression levels were observed in $11(27.5 \%)$ and $29(72.5 \%)$ patients, respectively. Preserved and reduced E-cadherin expression was observed in 19 (47.5\%) and 21 (52.5\%) patients, respectively. Patients with low Snail expression and preserved E-cadherin expression had a significantly lower risk of vascular invasion, lymphatic invasion, lymph node metastasis and liver metastasis and a lower WHO classification, as compared with the group that included patients with high Snail and reduced E-cadherin, high Snail and preserved E-cadherin, and low Snail and reduced E-cadherin expression. In addition, the patients with low Snail expression levels and preserved E-cadherin expression had more a favorable prognosis compared with the other group. The present study indicates that EMT serves an important role in tumor
\end{abstract}

Correspondence to: Dr Hiroshi Kurahara, Department of Digestive Surgery, Breast and Thyroid Surgery, Graduate School of Medical Sciences, Kagoshima University, 8-35-1 Sakuragaoka, Kagoshima 890-8520, Japan

E-mail: h-krhr@m3.kufm.kagoshima-u.ac.jp

Key words: cadherins, epithelial-mesenchymal transition, pancreatic neuroendocrine tumors progression in PNETs. Immunohistochemical evaluation of Snail and E-cadherin is useful for predicting the risk of vessel invasion and metastasis in PNETs.

\section{Introduction}

Pancreatic neuroendocrine tumors (PNETs) are a rare type of malignancy with an annual incidence of $1 / 100,000$ people, and account for $\sim 1-2 \%$ of all pancreatic tumors (1). PNETs are divided into functional tumors, which cause specific hormonal syndromes such as hypoglycemic attack, and non-functional tumors (2). The prognosis of PNETs is relatively favorable compared with that for pancreatic adenocarcinoma; however, certain patients with PNETs exhibit distant metastasis and have a less favorable prognosis (3). In 2010, the World Health Organization (WHO) categorized PNETs into G1 and G2 PNETs and neuroendocrine carcinoma (NEC), based on mitotic counts and the Ki-67 index (4). These classifications are useful for predicting prognosis and postoperative recurrence $(5,6)$. However, certain patients with low-risk pathologic features may unexpectedly experience distant metastasis and postsurgical recurrence (5). Little is currently known about the underlying molecular mechanisms by which distant metastasis is promoted in patients with PNETs.

Invasion into the surrounding normal tissue is a critical step for primary tumors to metastasize to distant areas (7). Recent studies have reported that the epithelial-mesenchymal transition (EMT) serves an important role in tumor progression and metastasis (8). Through EMT, cancer cells acquire the ability to migrate and infiltrate through the extracellular matrix, which leads to distant metastasis (7). At the molecular level, the transcriptional reprogramming of EMT is triggered by the transcription factors Snail, Slug and Twist, leading to subsequent suppression of specific adhesion molecules, including E-cadherin (9). E-cadherin is expressed in epithelial cells and performs an essential role in cell-cell contact (10). The expression of E-cadherin is decreased during EMT in embryonic development, tissue fibrosis and cancer (10). Snail was initially identified in Drosophila as the zinc-finger transcriptional repressor that binds to the E-boxes of the E-cadherin promoter $(11,12)$. Snail is able to effectively induce EMT via suppressing the transcription of E-cadherin during tumor progression (13). The loss of E-cadherin expression 
promotes Wnt signaling, and is associated with high levels of Snail in the nucleus (14). It was reported that high expression levels of Snail and low expression levels of E-cadherin are inversely correlated with the prognosis of patients with breast cancer (14). With the aim of gaining insight into the underlying molecular alterations in metastatic PNETs, the present study focused on EMT by evaluating Snail and E-cadherin expression. The significance of Snail and E-cadherin expression patterns in distant metastasis and prognosis was investigated in patients who had received surgery for PNETs.

\section{Materials and methods}

Patients and tumor samples. Formalin-fixed paraffinembedded blocks were obtained, containing tissue samples from 40 patients with histologically proven PNETs and who underwent surgical resection at Kagoshima University Hospital (Kagoshima, Japan) between January 1995 and December 2015. All resected tissue specimens were histologically examined using hematoxylin and eosin staining according to the tumor-node-metastasis classification and WHO classification 2010 systems $(4,15)$. The present study was approved by the Institutional Ethics Review Board of Kagoshima University Hospital, and written informed consent was obtained from all patients.

Antibodies. The rabbit anti-human polyclonal antibody directed against Snail (catalogue no. ab85936; Abcam, Cambridge, UK) was diluted at 1:500. The mouse anti-human monoclonal antibody against E-cadherin (catalogue no. M361229-2; Dako; Agilent Technologies, Inc., Santa Clara, CA, USA) was diluted at 1:500.

Immunohistochemical staining. All specimens were fixed in $10 \%$ formalin at room temperature and processed routinely. Sections ( $3 \mu \mathrm{m}$-thick) were cut from the paraffin blocks of primary tumors. Following deparaffinization in xylene and rehydration in graded solutions of ethanol, endogenous peroxidase activity was blocked by immersing the slides in absolute methanol solution containing $3 \%$ hydrogen peroxide for $30 \mathrm{~min}$ at room temperature. The sections were then treated with $1 \%$ goat serum albumin for Snail (catalogue no. S-1000) or $1 \%$ horse serum albumin for E-cadherin (catalogue no. S-2000) (both Vector Laboratories, Inc., Burlingame, CA, USA) for $30 \mathrm{~min}$ at room temperature to block nonspecific reactions. Heat-induced antigen retrieval via autoclave pretreatment $\left(120^{\circ} \mathrm{C}\right.$ for $\left.5 \mathrm{~min}\right)$ in citrate buffer solution ( $\mathrm{pH}$ 6.0) was performed. The sections were rinsed with PBS and incubated with the Snail antibody (dilution, 1:500) for $180 \mathrm{~min}$ and the E-cadherin antibody (dilution, 1:500) for $60 \mathrm{~min}$ at room temperature. Following incubation, the specimens were visualized using an Vectastain Elite ABC IgG Rabbit (catalogue no. PK-6101) or Mouse (catalogue no. PK-6102) kits (both Vector Laboratories, Inc.) and the Liquid DAB+ Substrate Chromogen system (catalogue no. K3468; Dako; Agilent Technologies, Inc.), according to the manufacturer's protocol. The slides were counterstained with hematoxylin prior to mounting. All reactions were performed with positive controls (mouse heart tissue for Snail and normal pancreatic tissue for E-cadherin). For the negative control, the primary antibody was replaced with PBS. No significant staining was observed in the negative-control sections.
Table I. Clinicopathological parameters of pancreatic neuroendocrine tumors $(n=40)$.

\begin{tabular}{|c|c|}
\hline Characteristics & $\mathrm{n}(\%)$ \\
\hline \multicolumn{2}{|l|}{ Age, years } \\
\hline$>60$ & $17(42.5)$ \\
\hline$\leq 60$ & $23(57.5)$ \\
\hline \multicolumn{2}{|l|}{ Gender } \\
\hline Male & $17(42.5)$ \\
\hline Female & $23(57.5)$ \\
\hline \multicolumn{2}{|l|}{ Tumor size } \\
\hline$>20 \mathrm{~mm}$ & $13(32.5)$ \\
\hline$\leq 20 \mathrm{~mm}$ & $27(67.5)$ \\
\hline \multicolumn{2}{|c|}{ Lymph node metastasis } \\
\hline No & $33(82.5)$ \\
\hline Yes & $7(17.5)$ \\
\hline \multicolumn{2}{|l|}{ Liver metastasis } \\
\hline No & $31(77.5)$ \\
\hline Yes & $9(22.5)$ \\
\hline \multicolumn{2}{|c|}{ WHO classification } \\
\hline G1 & $22(55.0)$ \\
\hline G2 & $15(37.5)$ \\
\hline $\mathrm{NEC}$ & $3(7.5)$ \\
\hline \multicolumn{2}{|l|}{ Vascular invasion } \\
\hline No & $24(60.0)$ \\
\hline Yes & $16(40.0)$ \\
\hline \multicolumn{2}{|c|}{ Lymphatic invasion } \\
\hline No & $28(70.0)$ \\
\hline Yes & $12(30.0)$ \\
\hline \multicolumn{2}{|l|}{ Functionality } \\
\hline Functioning & $23(57.5)$ \\
\hline Inslinoma & $15(37.5)$ \\
\hline Glucagonoma & $4(10.0)$ \\
\hline Gastrinoma & $4(10.0)$ \\
\hline Nonfunctioning & $17(42.5)$ \\
\hline
\end{tabular}

WHO, World Health Organization; NEC, neuroendocrine carcinoma.

Evaluation of immunohistochemistry. All tissue sections were simultaneously assessed by two investigators who were blinded to the patient clinicopathological data. Each slice was observed under an Olympus CX31 optical microscope (Olympus Corporation, Tokyo, Japan) (magnification, x400). Stained cells were assessed and quantified in five randomly selected fields. The immunohistochemical staining of Snail was classified as high expression if $\geq 10 \%$ of neoplastic cells exhibited nuclear staining, or as low expression if $<10 \%$ of neoplastic cells were stained. The expression of E-cadherin was compared between tumor cells and normal islet cells located adjacent to the tumor. Tumor cells that exhibited equivocal staining to normal islet cells were considered to have preserved expression of E-cadherin, whereas those that exhibited less intense staining patterns compared with the normal islet cells, or those that did 
A

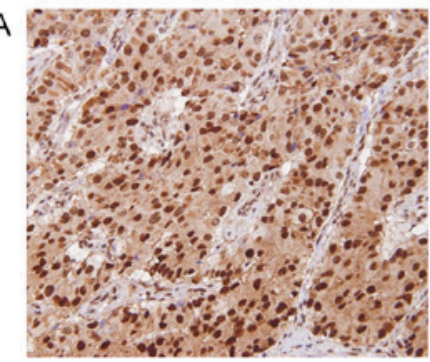

D

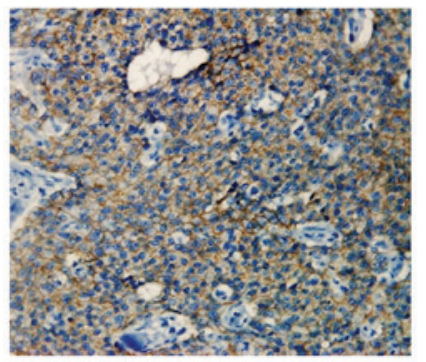

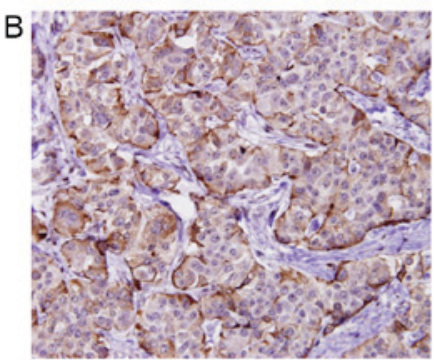

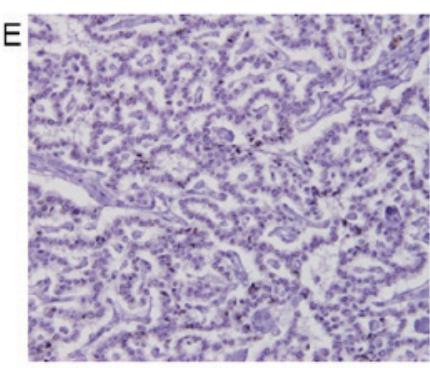

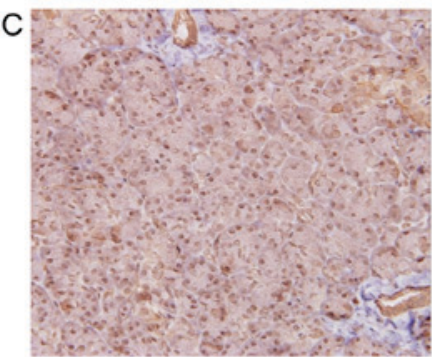

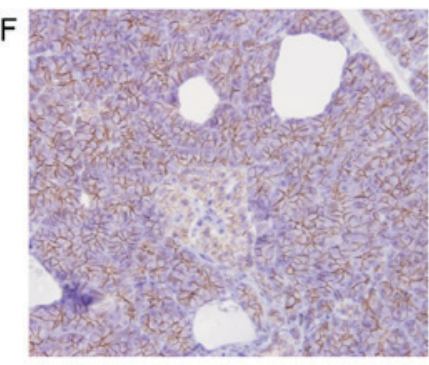

Figure 1. Representative images of (A-C) immunohistochemical Snail and (D-F) E-cadherin expression in pancreatic neuroendocrine tumors and normal pancreatic tissues. (A) High levels of nuclear staining of Snail in pancreatic neuroendocrine tumor. (B) Low levels of nuclear staining of Snail in pancreatic neuroendocrine tumor. (C) Snail expression in normal pancreatic tissue. (D) Preserved levels of membrane staining of E-cadherin in pancreatic neuroendocrine tumor. (E) Reduced levels of membrane staining of E-cadherin in pancreatic neuroendocrine tumor. (F) E-cadherin expression in normal pancreatic tissue. All images were captured at magnification, x400.

not stain at all, were considered to have reduced expression of E-cadherin.

Statistical analysis. Associations between different categorical variables were assessed using Fisher's exact test and the $\chi^{2}$ test. The Kaplan-Meier method was used for survival analysis, and variations in survival rates were estimated using the log-rank test. All statistical analysis was performed using SPSS version 22.0 (IBM SPSS, Armonk, NY, USA). All tests were two-sided. $\mathrm{P}<0.05$ was considered to indicate a statistically significant difference.

\section{Results}

Patient characteristics. Table I lists the clinicopathological features of the patients enrolled in the present study. The study group comprised 17 men and 23 women with an age range between 18 and 79 years (mean, 56.1 years). Lymph node metastasis was observed in 7 patients $(17.5 \%)$. Distant metastasis was observed in 9 patients $(22.5 \%)$, and the site of metastasis was the liver. Postoperative recurrence or an increase in the residual tumor size was observed in $10(25.0 \%)$ patients.

Expression of Snail and E-cadherin in PNETs. Fig. 1 depicts representative images of Snail and E-cadherin expression. Snail expression was identified in the nucleus of neoplastic cells. The proportion of immunoreactive cells varied, ranging from 0 to $>50 \%$ of neoplastic cells. The expression of E-cadherin in neoplastic cells was heterogeneous, ranging from 0 to $\sim 100 \%$ of neoplastic cells. High and low Snail expression levels were observed in 11 (27.5\%) and 29 (72.5\%) patients, respectively. Preserved and reduced E-cadherin expression was observed in $19(47.5 \%)$ and 21 (52.5) patients, respectively.
Association between Snail and E-cadherin expression and clinicopathological features. Table II demonstrates the association between Snail/E-cadherin expression and various clinicopathological factors. High Snail expression levels were significantly associated with high incidences of lymphatic and vascular invasion and liver metastasis $(\mathrm{P}=0.0078, \mathrm{P}<0.0001$ and $\mathrm{P}=0.0067$, respectively; Table II). Reduced E-cadherin expression levels were also significantly associated with high incidences of lymphatic and vascular invasion and liver metastasis $(\mathrm{P}=0.0015, \mathrm{P}=0.0270$ and $\mathrm{P}=0.0214$, respectively; Table II). No significant difference was observed in Snail and E-cadherin expression according to gender, tumor size and tumor functionality. Furthermore, on the basis of the expression profiles of Snail and E-cadherin, all patients were divided into two groups as follows: Low Snail and preserved E-cadherin expression, and the 'other group' (patients with high Snail and reduced E-cadherin, high Snail and preserved E-cadherin, and low Snail and reduced E-cadherin expression) (Table III). Patients in the 'other group' experienced a markedly increased lymph node metastasis, liver metastasis, G2 and NEC in the WHO classification, vascular invasion and lymphatic invasion, as compared with in the low Snail and preserved E-cadherin expression group. Patients with low Snail and preserved E-cadherin expression were not observed to exhibit lymph node metastasis or liver metastasis during the present study. In total, 1/22 patients with a G1 classified tumor experience liver metastasis; these particular tumor tissues exhibited high Snail and reduced E-cadherin expression levels.

Prognostic impact of Snail and E-cadherin expression. The 5-year and 10-year survival rates for the patient cohort were determined to be 91.7 and $59.5 \%$, respectively. Fig. 2 presents the overall survival (OS) and progression-free survival (PFS) rates following surgery in the aforementioned two groups, based on the expression levels of Snail and E-cadherin. The 
Table II. Association between the expression of Snail/E-cadherin and clinicopathological factors in pancreatic neuroendocrine tumors.

\begin{tabular}{|c|c|c|c|c|c|c|c|}
\hline \multirow[b]{2}{*}{ Characteristics } & \multirow[b]{2}{*}{ Total, $\mathrm{n}$} & \multicolumn{2}{|c|}{ Snail expression, $\mathrm{n}$} & \multirow[b]{2}{*}{ P-value } & \multicolumn{2}{|c|}{ E-cadherin expression, $\mathrm{n}$} & \multirow[b]{2}{*}{ P-value } \\
\hline & & Low & High & & Reduced & Preserved & \\
\hline Total & 40 & 29 & 11 & & 21 & 19 & \\
\hline Age, years $($ mean \pm SD) & & $56.0 \pm 3.20$ & $56.4 \pm 4.46$ & NS & $59.4 \pm 3.62$ & $52.5 \pm 3.63$ & NS \\
\hline Gender & & & & NS & & & NS \\
\hline Male & 17 & 11 & 6 & & 9 & 8 & \\
\hline Female & 23 & 18 & 5 & & 12 & 11 & \\
\hline Tumor size & & & & NS & & & NS \\
\hline$>20 \mathrm{~mm}$ & 13 & 9 & 4 & & 9 & 4 & \\
\hline$\leq 20 \mathrm{~mm}$ & 27 & 20 & 7 & & 12 & 15 & \\
\hline Lymph node metastasis & & & & & & & NS \\
\hline No & 33 & 26 & 7 & & 15 & 18 & \\
\hline Yes & 7 & 3 & 4 & NS & 6 & 1 & \\
\hline Liver metastasis & & & & 0.0067 & & & 0.0214 \\
\hline No & 31 & 26 & 5 & & 13 & 18 & \\
\hline Yes & 9 & 3 & 6 & & 8 & 1 & \\
\hline WHO classification & & & & NS & & & NS \\
\hline G1 & 22 & 18 & 4 & & 9 & 13 & \\
\hline $\mathrm{G} 2+\mathrm{NEC}$ & 18 & 11 & 7 & & 12 & 6 & \\
\hline Vascular invasion & & & & $<0.0001$ & & & 0.0270 \\
\hline No & 24 & 23 & 1 & & 9 & 15 & \\
\hline Yes & 16 & 6 & 10 & & 12 & 4 & \\
\hline Lymphatic invasion & & & & 0.0078 & & & 0.0015 \\
\hline No & 28 & 24 & 4 & & 10 & 18 & \\
\hline Yes & 12 & 5 & 7 & & 11 & 1 & \\
\hline Functionality & & & & NS & & & NS \\
\hline Functioning & 23 & 18 & 5 & & 12 & 11 & \\
\hline Nonfunctioning & 17 & 11 & 6 & & 9 & 8 & \\
\hline
\end{tabular}

NS, not significant; WHO, World Health Organization; NEC, neuroendocrine carcinoma.

'other group' exhibited a poorer OS rate, as compared with the low Snail and preserved E-cadherin expression group, although these differences were not statistically significant (Fig. 2A). By contrast, a significant difference was observed between the two groups with regard to the rate of PFS ( $\mathrm{P}=0.0099$; Fig. 2B).

\section{Discussion}

The present study demonstrated that the analysis of Snail and E-cadherin co-expression may be useful for predicting vascular invasion, lymphatic invasion and liver metastasis in patients with PNETs. The PNET tissue samples in the present study were divided into the following two groups: Tumors with low Snail and preserved E-cadherin expression, and the other tumors which consisted of any tumor tissues with high Snail and reduced E-cadherin, high Snail and preserved E-cadherin, and low Snail and reduced E-cadherin expression. Tumors in the low Snail and preserved E-cadherin expression group and in the 'other group' were considered to have epithelial and mesenchymal phenotypes, respectively. PNETs with a mesenchymal phenotype exhibited a significantly higher frequency of vascular and lymphatic invasion, lymph node metastasis and liver metastasis. Even in G2 tumors and NEC, those with low Snail and preserved E-cadherin expression did not have lymph node and liver metastasis. In PNETs, liver metastasis is the most frequent mode of recurrence and a significant adverse prognostic factor $(16,17)$. Our study indicated that EMT serves an important role in lymphatic invasion, vascular invasion, lymph node metastasis and liver metastasis in PNETs. Immunohistochemical evaluations of the EMT markers Snail and E-cadherin were useful for predicting metastasis.

It has been reported that EMT performs a fundamental role in tumor invasion and metastasis in various types of cancer. In pancreatic adenocarcinoma, numerous previous studies have revealed that Snail, zinc finger E-box-binding homeobox (ZEB) 1, ZEB2 and nectins are associated with EMT and with poor prognosis (18-20). However, the role of EMT in 
Table III. Association between the expression of Snail and E-cadherin and clinicopathological factors in pancreatic neuroendocrine tumors.

Snail and E-cadherin expression, $\mathrm{n}$

\section{Characteristic}

Total

Age, years (mean \pm SD)

Gender

Male

Female

Tumor size

$>20 \mathrm{~mm}$

$\leq 20 \mathrm{~mm}$

Lymph node metastasis

No

Yes

Liver metastasis

$\begin{array}{lr}\text { No } & 31 \\ \text { Yes } & 9\end{array}$

WHO classification

$\begin{array}{ll}\text { G1 } & 22 \\ \mathrm{G} 2+\mathrm{NEC} & 18\end{array}$

Vascular invasion

No

Yes

Lymphatic invasion

No

Yes

Functionality

Functioning

Nonfunctioning

40

17

23

13

27

33

7

31

22

Total Snail low + E-cadherin preserved

9

18

16

$51.8 \pm 4.23$

6

10

Other group ${ }^{\mathrm{a}}$

P-value

24

$59.0 \pm 3.22$

NS

NS

11

13

$4 \quad 9$

$12 \quad 15$

$16 \quad 17$

$\begin{array}{ll}0 & 7\end{array}$

$16-15$

0

$10 \quad 12$

$6 \quad 12$

$15 \quad 9$

$1+15$

$16 \quad 12$

$0 \quad 12$

$23 \quad 10 \quad 13$

$17 \quad 6 \quad 11$

a'Other group' included patients with high Snail and reduced E-cadherin, high Snail and preserved E-cadherin, and low Snail and reduced E-cadherin expression. NS, not significant; WHO, World Health Organization; NEC, neuroendocrine carcinoma.

A Overall survival

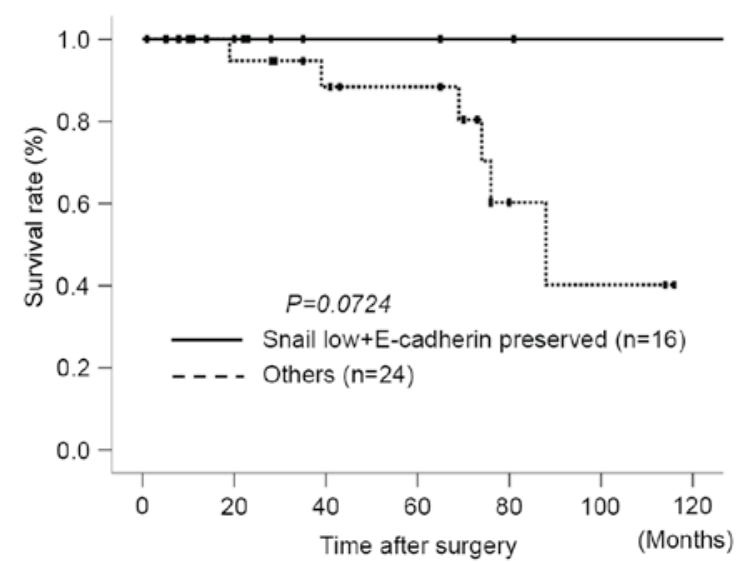

B Progression-free survival

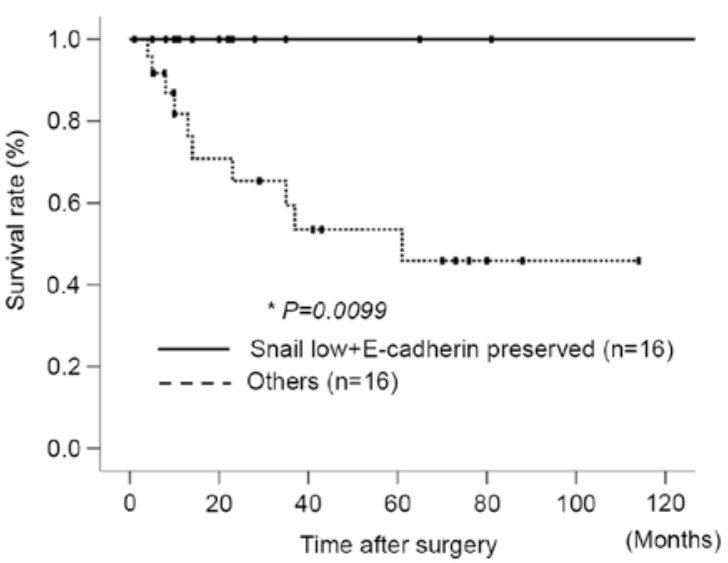

Figure 2. Kaplan-Meier survival curves for patients with pancreatic neuroendocrine tumors who underwent surgical resection. All patients were divided into two groups: The group with low Snail and preserved E-cadherin expression, and the 'other group' that included patients with high Snail and reduced E-cadherin, high Snail and preserved E-cadherin, and low Snail and reduced E-cadherin expression. (A) No significant difference in the overall survival rate was observed between the two groups. (B) A significant difference in the progression-free survival rate was observed between the two groups. 
PNETs is still unclear. Fendrich et al (21) immunohistochemically evaluated the expression patterns of the EMT markers E-cadherin, Snail and Twist in human PNETs, and observed a loss of E-cadherin and the overexpression of Snail and Twist in the majority of malignant PNETs. In addition, the formation of islet cell tumors in the RIP-Tag2 transgenic mouse model was prevented by inhibiting Snail expression using polyethylene glycol. This result indicated that EMT serves a key role in the tumorigenesis of PNETs. Galvan et al (22) also used immunohistochemistry to assess the expression of EMT markers in 91 cases of gastroenteropancreatic (GEP) NET, including 22 cases of PNET. It was reported that alteration of the E-cadherin/ $\beta$-catenin complex, specifically the high Snail expression and cytoplasmic E-cadherin pattern, reduced the survival rates of patients with GEP-NETs. Taken together, the current study and previous reports on PNETs have demonstrated that tumor cells that have acquired an invasive phenotype through EMT may easily detach from the primary tumor, invade surrounding tissues and enter microvessels, therefore spreading into the circulation $(21,22)$.

In the present study, the expression of Snail and E-cadherin was not significantly associated with the rate of OS. This is partly as the recurrence of PNETs, which most commonly involves liver metastasis, is usually treatable by transcatheter arterial chemoembolization or transcatheter arterial infusion, and then effectively controlled (23-25). The current study had various limitations; the study design was retrospective, and the patient cohort was small. However, the expression patterns of Snail and E-cadherin were identified to be potent predictors of lymph node and liver metastasis. To the best of our knowledge, this is the first study to demonstrate that EMT may perform an important role in the spread of tumor cells into the lymphatic flow and circulation, and in the subsequent establishment of metastasis in PNETs. Additional studies, with larger patient cohorts are required to verify the results of the present study.

In conclusion, the current study demonstrated that EMT may serve an important role in vessel invasion and metastasis in PNETs, and that the immunohistochemical evaluation of Snail and E-cadherin expression is useful for predicting the invasive and metastatic phenotype of PNETs.

\section{Acknowledgements}

The authors would like to thank Enago (www.enago.jp) for reviewing the English language of the original manuscript.

\section{References}

1. Oberg K and Eriksson B: Endocrine tumours of the pancreas. Best Pract Res Clin Gastroenterol 19: 753-781, 2005.

2. Klöppel G, Rindi G, Anlauf M, Perren A and Komminoth P: Site-specific biology and pathology of gastroenteropancreatic neuroendocrine tumors. Virchows Arch 451 (Suppl 1): S9-S27, 2007.

3. Metz DC and Jensen RT: Gastrointestinal neuroendocrine tumors: Pancreatic endocrine tumors. Gastroenterology 135: 1469-1492, 2008.

4. Bosman FT, Carneiro F, Hruban R and Theise N: WHO Classification of Tumours of the Digestive System. Vol 3. 4th edition. IARC Press, 2010.

5. Fischer L, Bergmann F, Schimmack S, Hinz U, Prieß S, Müller-Stich BP, Werner J, Hackert T and Büchler MW: Outcome of surgery for pancreatic neuroendocrine neoplasms. Br J Surg 101: 1405-1412, 2014.
6. Tsutsumi K, Ohtsuka T, Fujino M, Nakashima H, Aishima S, Ueda J, Takahata S, Nakamura M, Oda Y and Tanaka M: Analysis of risk factors for recurrence after curative resection of well-differentiated pancreatic neuroendocrine tumors based on the new grading classification. J Hepatobiliary Pancreat Sci 21: 418-425, 2014.

7. Jiang JH, Liu C, Cheng H, Lu Y, Qin Y, Xu YF, Xu J, Long J, Liu L, Ni QX and Yu XJ: Epithelial-mesenchymal transition in pancreatic cancer: Is it a clinically significant factor? Biochim Biophys Acta 1855: 43-49, 2015.

8. Kalluri R and Weinberg RA: The basics of epithelial-mesenchymal transition. J Clin Invest 119: 1420-1428, 2009.

9. Maeda M, Johnson KR and Wheelock MJ: Cadherin switching: Essential for behavioral but not morphological changes during an epithelium-to-mesenchyme transition. J Cell Sci 118: 873-887, 2005

10. Gunji N, Oda T, Todoroki T, Kanazawa N, Kawamoto T, Yuzawa K, Scarpa A and Fukao K: Pancreatic carcinoma: Correlation between E-cadherin and alpha-catenin expression status and liver metastasis. Cancer 82: 1649-1656, 1998.

11. Nieto MA: The snail superfamily of zinc-finger transcription factors. Nat Rev Mol Cell Biol 3: 155-166, 2002.

12. Thomson S, Buck E, Petti F, Griffin G, Brown E, Ramnarine N, Iwata KK, Gibson N and Haley JD: Epithelial to mesenchymal transition is a determinant of sensitivity of non-small-cell lung carcinoma cell lines and xenografts to epidermal growth factor receptor inhibition. Cancer Res 65: 9455-9462, 2005.

13. Carver EA, Jiang R, Lan Y, Oram KF and Gridley T: The mouse snail gene encodes a key regulator of the epithelial-mesenchymal transition. Mol Cell Biol 21: 8184-8188, 2001.

14. Blanco MJ, Moreno-Bueno G, Sarrio D, Locascio A, Cano A, Palacios J and Nieto MA: Correlation of Snail expression with histological grade and lymph node status in breast carcinomas. Oncogene 21: 3241-3246, 2002.

15. Rindi G, Klöppel G, Alhman H, Caplin M, Couvelard A, de Herder WW, Erikssson B, Falchetti A, Falconi M, Komminoth $\mathrm{P}$, et al: TNM staging of foregut (neuro)endocrine tumors: A consensus proposal including a grading system. Virchows Arch 449: 395-401, 2006.

16. Birnbaum DJ, Turrini O, Vigano L, Russolillo N, Autret A, Moutardier V, Capussotti L, Le Treut YP, Delpero JR and Hardwigsen J: Surgical management of advanced pancreatic neuroendocrine tumors: Short-term and long-term results from an international multi-institutional study. Ann Surg Oncol 22: 1000-1007, 2015

17. Saeed A, Buell JF and Kandil E: Surgical treatment of liver metastases in patients with neuroendocrine tumors. Ann Transl Med 1: 6, 2013 .

18. Hotz B, Arndt M, Dullat S, Bhargava S, Buhr HJ and Hotz HG: Epithelial to mesenchymal transition: Expression of the regulators snail, slug, and twist in pancreatic cancer. Clin Cancer Res 13: 4769-4776, 2007.

19. Kurahara H, Takao S, Maemura K, Mataki Y, Kuwahata T, Maeda K, Ding Q, Sakoda M, Iino S, Ishigami S, et al: Epithelial-mesenchymal transition and mesenchymal-epithelial transition via regulation of ZEB-1 and ZEB-2 expression in pancreatic cancer. J Surg Oncol 105: 655-661, 2012.

20. Izumi H, Hirabayashi K, Nakamura $\mathrm{N}$ and Nakagohri T: Nectin expression in pancreatic adenocarcinoma: Nectin-3 is associated with a poor prognosis. Surg Today 45: 487-494, 2015.

21. Fendrich V, Maschuw K, Waldmann J, Buchholz M, Rehm J, Gress TM, Bartsch DK and König A: Epithelial-mesenchymal transition is a critical step in tumorgenesis of pancreatic neuroendocrine tumors. Cancers (Basel) 4: 281-294, 2012.

22. Galvan JA, Astudillo A, Vallina A, Fonseca PJ, Gómez-Izquierdo L, García-Carbonero R and González MV: Epithelial-mesenchymal transition markers in the differential diagnosis of gastroenteropancreatic neuroendocrine tumors. Am J Clin Pathol 140: 61-72, 2013.

23. Therasse E, Breittmayer F, Roche A, De Baere T, Indushekar S, Ducreux M, Lasser P, Elias D and Rougier P: Transcatheter chemoembolization of progressive carcinoid liver metastasis. Radiology 189: 541-547,1993.

24. Ruszniewski P, Rougier P, Roche A, Legmann P, Sibert A, Hochlaf S, Ychou M and Mignon M: Hepatic arterial chemoembolization in patients with liver metastases of endocrine tumors. A prospective phase II study in 24 patients. Cancer 71: 2624-2630, 1993.

25. Lee E, Leon Pachter H and Sarpel U: Hepatic arterial embolization for the treatment of metastatic neuroendocrine tumors. Int J Hepatol 2012: 471203, 2012. 